\title{
CURSO DE ESPECIALIZAÇÃO DE ENFERMAGEM EM UNIDADE DE TERAPIA INTENSIVA
}

\author{
Moema Guedes Barbato * \\ Clarinda Takito * * \\ 1 - INTRODUÇÃO
}

O avanço da tecnologia e pesquisa no campo da assistência à saúde solicita pessoal devidamente qualificado.

A natureza especializada da assistência ao paciente, ensino e pesquisa em uma Unidade de Terapia Intensiva influenciam marcadamente o atendimento e atividade do pessoal de enfermagem. Isto inclui desde a realização de tarefas de rotina até procedimentos que variam em dificuldade e complexidade, demandando alto grau de experiência técnico-científica e conhecimentos teóricos de vários campos da ciência.

A participação efetiva dos profissionais de enfermagem no trabalho da equipe de saúde tem êxito quando há continuidade e homegeneidade. Esses comportamentos são observados se os elementos estão técnica e cientificamente preparados; neste caso o inter-relacionamento transcorre de maneira harmônica e objetiva. Isto se faz sentir, notadamente em uma U.T.I., sistema complexo, que tem como objetivo tratar de pacientes criticamente doentes $\mathrm{e}$ diminuir o índice de mortalidade por complicações, através de assistência permanente.

$O$ desempenho da função da enfermagem em assistir o paciente de maneira que este recupere total ou parcialmente suas funções bio-psico-sociais, requer capacidade de análise, discernimento, bem como ação autônoma, fundamentada em princípios e conhecimentos cien. tíficos.

( ${ }^{*}$ ) Auxiliar de Ensino do Departamento de Orientação Profissional da Escola de Enfermagem da U.S.P.

$\left({ }^{\star \star}\right)$ Enfermeira do Hospital das Clínicas de São Paulo respectivamente. 
A habilidade de estabelecer e implementar um curso de ação diante do problema de um paciente numa situação de emergência, dependerá dos conhecimentos técnicos, científicos e humanos que a enfermeira possuir.

Em face a essa necessidade foi criado o primeiro curso de especialização de enfermagem em Unidade de Terapia Intensiva. O planejamento e a realização do curso foram feitos por elementos da Escola de Enfermagem da Universidade de São Paulo - (EEUSP), Associação Brasileira de Enfermagem - Seção de São Paulo (ABEn-SP), Hospital das Clínicas da Faculdade de Medicina da Universidade de São Paulo - (HC-FMUSP), Hospital do Servidor Público do Estado - HSPE), representados em comissão por D. Maria Rosa Pinheiro, diretora da EEUSP, Moema Guedes Barbato, professora da EEUSP e coordenadora do curso, Lourdes Torres de Cerqueira, presidente da ABEn-SP, Dr. Luís Olímpio Teixeira Nascimento, representante do Corpo Clínico do HC, Lourdes Muller, representante do Corpo de Enfermagem do HC, e Agmar Resende, representante do Corpo de Enfermagem do HSPE. Esta comissão instituiu um regimento, estabelecendo convênio com o Ministério da Educação e Cultura (MEC), através do órgão Programas Intensivos de Preparo de Mão-de-Obra (PIPMO), que forneceu condições financeiras para realização do referido curso.

O Hospital das Clínicas e o Hospital do Servidor Público do Estado serviram de campo de estágło.

\section{2 - PlaneJAMENTO DO CURSO}

\section{1 - Objetivos do Curso}

A pesquisa preliminar, através de observação sistemática das Unidades de Terapia Intensiva, forneceu elementos significativos: número de UTIs nos hospitais selecionados para estágio; especialidades; número de pacientes em cada unidade; incidência de casos; planta física da unidade; e, principalmente funções exercidas pelas enfermeiras.

Partindo desses dados foram determinados os objetivos do curso - fornecer à enfermeira a capacidade de:

a) discorrer sobre os aspectos clínicos (etiologia, sinais e sintomas), tratamentos médico e cirúrgicos, exames complementares, e assistência específica e integral de enfermagem dos pacientes admitidos nas UTIs; dades;

b) manejar tecnicamente aparelhos e equipamentos das uni-

c) planejar, organizar, administrar e assessorar uma UTI. 
Esses objetivos gerais permitiram, posteriormente, traçar as diretrizes programáticas.

\section{2 - Diretrizes Programáticas}

O programa do curso foi planejado de acordo com os objetivos gerais, levando em consideração as áreas de estágio disponíveis. Foram selecionadas as seguintes áreas:

- no HC:

- Unidade de Recuperação Pós-operatória geral;

- Unidade de Recuperação Pós-operatória de Cirurgia Cardíaca;

- Unidade de Cardiologia Clínica;

- Unidade de Choque;

- Unidade de Transplante Renal;

- Unidade de Hemo-Diálise;

- Unidade de Pós-operatório em Neurologia;

- Unidade de Politraumatizados;

- Unidade de Queimados;

- Unidade de Tétano;

- no HSPE:

- Unidade de Recuperação Cardíaca;

- Unidade de Terapia Intensiva Geral;

- Unidade Renal.

Foram elaboradas dez (10) unidades didáticas, abrangendo os seguintes aspectos:

a) anatomia, fisiopatologia, exames complementares, terapêutica e assistência de enfermagem aos pacientes acometidos de afecções agudas pulmonares, cardiovasculares, renais, neurológicas, endocrinológicas e hematológicas (seis unidades);

b) emergências com pacientes em choque, politraumatizados, queimados e com envenenamentos (duas unidades); dade);

c) problemas psico-sociais dos pacientes graves (uma uni-

d) administração de enfermagem em UTI (uma unidade). 
A partir desses trabalhos foi fixado o número de vinte e cinco (25) vagas, capacidade máxima das áreas de estágio. Admitiu-se uma sobrecarga de mais vinte e cinco (25) vagas para ouvintes no bloco teórico.

\section{3 - Carga Horária}

O curso foi planejado para um semestre acadêmico, correspondendo a dezesseis semanas, total de trezentas e trinta horas (vinte e dois créditos). As seis primeiras semanas foram dedicadas ao bloco teórico com uma carga de cento e cinco horas (sete créditos).

As unidades didáticas mais complexas foram programadas com duração de aproximadamente quinze (15) horas (afecções cardio-vasculares, pulmonares, renais e choque).

\section{4 - Avaliáção do Rendimento Escolar}

O critério de aprovação baseou-se no regimento do curso, seu instrumento básico. O aluno estará aprovado se conseguir nota final igual ou superior a cinco (5), média aritmética entre as notas dos trabalhos escritos e orais (média das notas do bloco teórico), e notas dos estágios semanais (média das notas da parte prática).

A supervisão dos estágios semanais foi programada para três enfermeiros: dois para cobrir as áreas do HC e um para o HSPE, para os quais foi estabelecido um roteiro de orientação quanto ao tipo de supervisão: forma de observação, ensino e avaliação (anexo I).

Seria reprovado o aluno com freqüência inferior a setenta e cinco por cento $(75 \%)$ das aulas teóricas e práticas (estágios) dadas

\section{5 - Corpo Docente e Areas de Estágio}

Para atingir os objetivos do curso, a comissão estabeleceu requisitos quanto ao corpo docente e áreas de estágio:

- o corpo docente, constituído por médicos e enfermeiras, deveria ser selecionado entre elementos com experiência na especialidade e possuir conhecimentos técnico-científicos e didáticos;

- as áreas de estágio devidamente montadas com aparelhos da especialidade (ventiladores, cardioscópios, desfibriladores, rim artificial, polígrafos, monitores, sistemas de oxigênio, vácuo e ar comprimido e outros); possuir boa equipe de trabalho e oferecer condições razoáveis de aprendizagem. 


\section{3 - EXECUÇÃO DO PLANO DO CURSO}

\section{1 - Bloco Teórico}

O bloco teórico constou de três aulas diárias, conforme programa estabelecido.

Foram preenchidas as 25 vagas criadas, e as ouvintes (25) participaram voluntariamente de discussões e testes.

As aulas teóricas foram dadas em forma expositiva e em discussão de grupo, com utilização de material audiovisual, complementadas com apostilas distribuídas no decorrer do curso.

Em algumas unidades, houve pré-testes para avaliação de comportamentos iniciais.

Durante o bloco teórico houve reuniões do corpo discente, docente e coordenadores, para avaliação do andamento do curso, estudos dos problemas e soluções sugeridas.

Ao término do bloco teórico foi realizado um painel com representantes de alguns centros de Terapia Intensiva e participação do plenário, onde foram discutidos assuntos relativos a funções $e$ atribuições da enfermeira, qualidade e quantidade de pessoal necessário para desenvolvimento do trabalho em UTI.

\section{2 - Parte Prática}

Numa reunião preliminar entre supervisoras, coordenação e as vinte e cinco alunas inscritas foram distribuídas as escalas de rodízio semanal, que permitiram à cada aluna estagiar em dez unidades diferentes nos dois hospitais, abrangendo todas as especialidades.

\subsection{1 - Programação Semanaal}

Cada supervisor reunia seu grupo às 2."s feiras para programação semanal das atividades individuais e em equipe, a ser desenvolvida na seguinte seqüência:

1. dia:

- apresentação das particularidades da unidade;

- apresentação da equipe de trabalho;

- verificação e leitura do Kardex;

- leitura de rotinas; 
— observação da dinâmica de trabalho;

- escolha de um paciente para elaboração de um plano de cuidados.

2. ${ }^{\circ}$ dia:

— observação dos pacientes;

- verificação dos prontuários;

- estudo do paciente escolhido;

- levantamento de dados, identificação de problemas, proposição de resoluções através de um plano de cuidados;

- execução do plano e avaliação geral com a supervisora.

\section{$3 .^{\bullet}$ dia :}

- igual ao dia anterior, relacionando o plano de cuidados às anotações e exames complementarẹs.

4. dia:

- igual aos dias anteriores, relacionando o plano de cuidados à terapêutica e patologia.

$50^{\circ}$ dia:

- livre para estudo e consultas;

- reunião do grupo para avaliação do estágio;

- apresentação de estudo de caso.

\subsection{2 - Supervisão}

Nas visitas que os supervisores faziam diariamente às unidades, tinham a oportunidade de entrevistar as estagiárias, verificar dificuldades, complementar conhecimentos, resolver problemas, e discutir com cada uma o plano de cuidado.

\subsection{3 - Plano de Cuidados}

Para exemplificar o tipo de plano exigido, onde o conteúdo sempre preocupou mais que a forma, segue o anexo II, realizado por uma aluna em estágio na Unidade de Transplante Renal. 


\subsection{4 - Estudo de Caso}

Os estudos de caso despertaram grande interesse nos grupos, que participaram ativamente das discussões e assimilaram grande parte do conteúdo discutido.

Foi usada a seguinte técnica:

30 minutos - exposição do quadro clínico, exames complementares e assistência de enfermagem.

15 minutos - evolução do quadro clínico, exames complementares e assistência de enfermagem.

45 minutos - discussão, conclusão e sugestões.

O tempo total da apresentação foi dividido em:

1/3 para quadro clínico e laboratorial;

2/3 para assistência de enfermagem.

Foi dada ênfase à função da enfermeira diante dos problemas que o paciente apresentava, situando o grupo em seu papel profissional de conhecimento e atuação.

Ao término da parte prática foi realizada uma prova de $\mathbf{1 2 0}$ testes, escolha única, sobre assuntos expostos no bloco teórico e nos estágios.

\section{4 - CONCLUSס̃ES}

O Curso de Especialização de Enfermagem em Unidade de Terapia Intensiva teve, na opinião da comissão e das alunas, um saldo altamente positivo.

\section{1 - Conclusão das Estudantes}

As avaliações escritas feitas pelas enfermeiras no final do curso, sobre toda a dinâmica do mesmo, bloco teórico e parte prática, apresentam críticas e sugestões que devem ser consideradas em cursos futuros desta natureza:

- estabelecer avaliações escritas após cada unidade didática e cada área de estágio;

- ater-se, dentro das aulas teóricas, ao assunto básico, sem detalhes demasiadamente especializados; 
- ampliar o tempo do bloco teórico mantendo o mesmo número de horas-aula;

- estabelecer contato preliminar entre a coordenação do curso e as enfermeiras operantes nas áreas de estágio;

- áreas de estágio consideradas em número suficiente e de boa qualidade;

- supervisão boa quanto à qualidade mas em quantidade insuficiente ( 8 a 9 alunas por supervisor abrangendo 3 a 4 áreas de estágio);

- plano de cuidados e estudo de casos considerados de boa aprendizagem.

\section{Sugestões:}

- maior número de supervisores;

- maior número de estudos de caso;

- horário integral para melhor aproveitamento do curso.

\section{2 - Conclusão das Autoras}

O Curso de Especialização de Enfermagem em UTI, o primeiro realizado no Estado de São Paulo, apresentou dificuldades principalmente com relação à seleção do corpo docente e áreas de estágio. Terapia Intensiva é um campo novo, com grande atividade de trabalho e pesquisa, onde os elementos atuantes, em pequeno número, tem pouca disponibilidade de tempo. Muitas das unidades estão em fase de crescimento, todas com pequeno número de leitos (um a seis pacientes), podendo acolher no máximo dois estagiários por vez.

Apesar destas e outras dificuldades encontradas no planejamento, foram satisfeitos os requisitos propostos pela comissão e atingidos os objetivos do curso.

Com a colaboração da comissão, da parte administrativa e de enfermagem dos hospitais e excelente receptividade das enfermeiras estudantes, foi feita uma distribuição de trabalhos satisfatória.

Receberam certificados 24 enfermeiras (uma afastou-se por motivo de doença) e quase todas estão dando seu trabalho em unidades de Terapia Intensiva.

\section{3 - Conclusão Geral}

O estudo e pesquisa em Terapia Intensiva, conjugado com o Interesse em seu aperfeiçoamento, foi a preocupação geral de cada 
enfermeira e do grupo. O profissional de enfermagem em Terapia Intensiva ocupa uma posição que o projeta profissionalmente, e em conseqüência a sua classe, a um nível de trabalho científico.

E necessário capacitar enfermeiras of erecendo conhecimentos e oportunidades de trabalho consciente. Só depois dessas condições básicas poderão ser desenvolvidas pesquisas que, além de proporcionar maior segurança ao paciente grave, irão enriquecer cada uma, mantendo a enfermagem num status de profissão liberal.

$O$ curso deve ser repetido anualmente, dando oportunidade para todas as enfermeiras que tiverem interesse em se especializar ou se atualizar na matéria. 


\section{A N E X O I \\ ORIENTAÇÃO A SUPERVISÃO}

I $-O$ supervisor deve:

1 - verificar a pontualidade e assiduidade de cada enfermeira;

2 - conferir horários e marcar o total de horas de cada dia; totalizar o número de horas/sem. e marcar na folha de avaliação;

3 - identificar as necessidades de conhecimentos e habilidades prioritárias de cada enfermeira, orientando-a no trabalho de campo;

4 - resolver problemas surgidos ou pedir assessoria à comissão para os casos de difícil solução;

5 - comunicar a uma das coordenadoras do curso o andamento do trabalho;

6 - preencher o boletim de avaliação semanal.

II - Como avaliar:

O supervisor deverá no final de cada estágio da enfermeira, avaliá-la nos conhecimentos, habilidades e atitudes demonstradas durante o trabalho.

$\mathrm{Na}$ área dos conhecimentos a aluna deve estar apta a:

1 - demonstrar conhecimentos científicos e aplicá-los nos cuidados de enfermagem; 
2 - identificar problemas biopsico-sociais dos pacientes e planejar soluções pertinentes;

3 - interpretar exames laboratoriais, radiológicos e traçados;

4 - citar os princípios farmacológicos das drogas ministradas.

$\mathrm{Na}$ área das habilidades:

1 - manejar os diversos aparelhos demonstrando reconhecer a aplicabilidade de cada um;

2 - identificar problemas dos pacientes e aplicar tratamentos específicos;

3 - dar assistência integral de enfermagem aos pacientes: cuidados físicos, psíquicos, sociais e espirituais;

4 - assistir à família, orientando-a sob diferentes aspectos: gravidade do caso, visitas, período hospitalar e alta;

5 - apresentar relatórios baseados em boas observações;

6 - apresentar o planejamento de estudos de caso e plano de cuidados.

$\mathrm{Na}$ área das atitudes a enfermeira deve:

1 - demonstrar iniciativa, pontualidade, assiduidade, controle emocional;

2 - apresentar espírito de colaboração à toda equipe;

3 - comunicar-se com eficiência;

4 - aplicar os conhecimentos deontológicos;

5 - demonstrar respeito e interesse pela pessoa humana. 


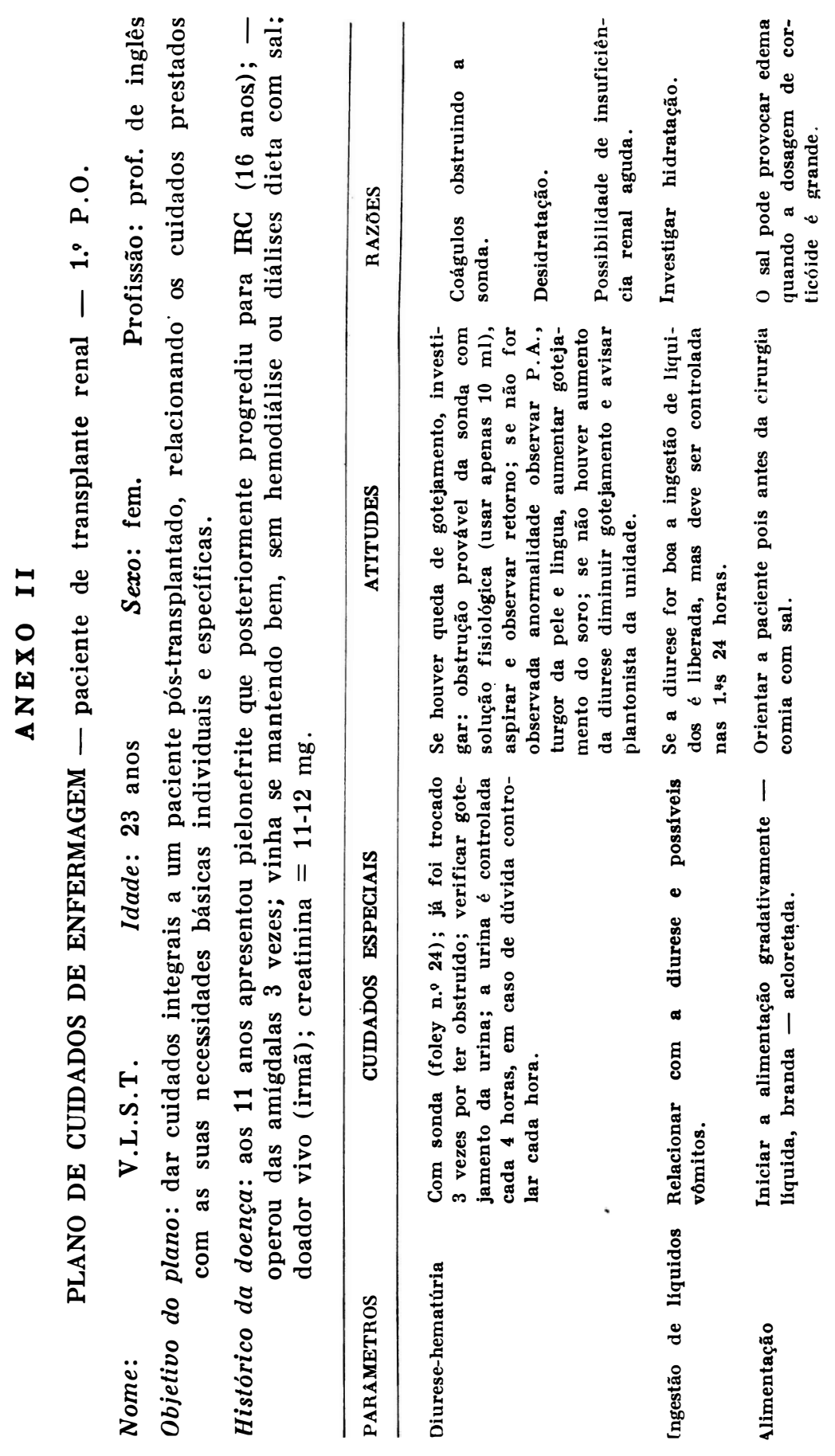




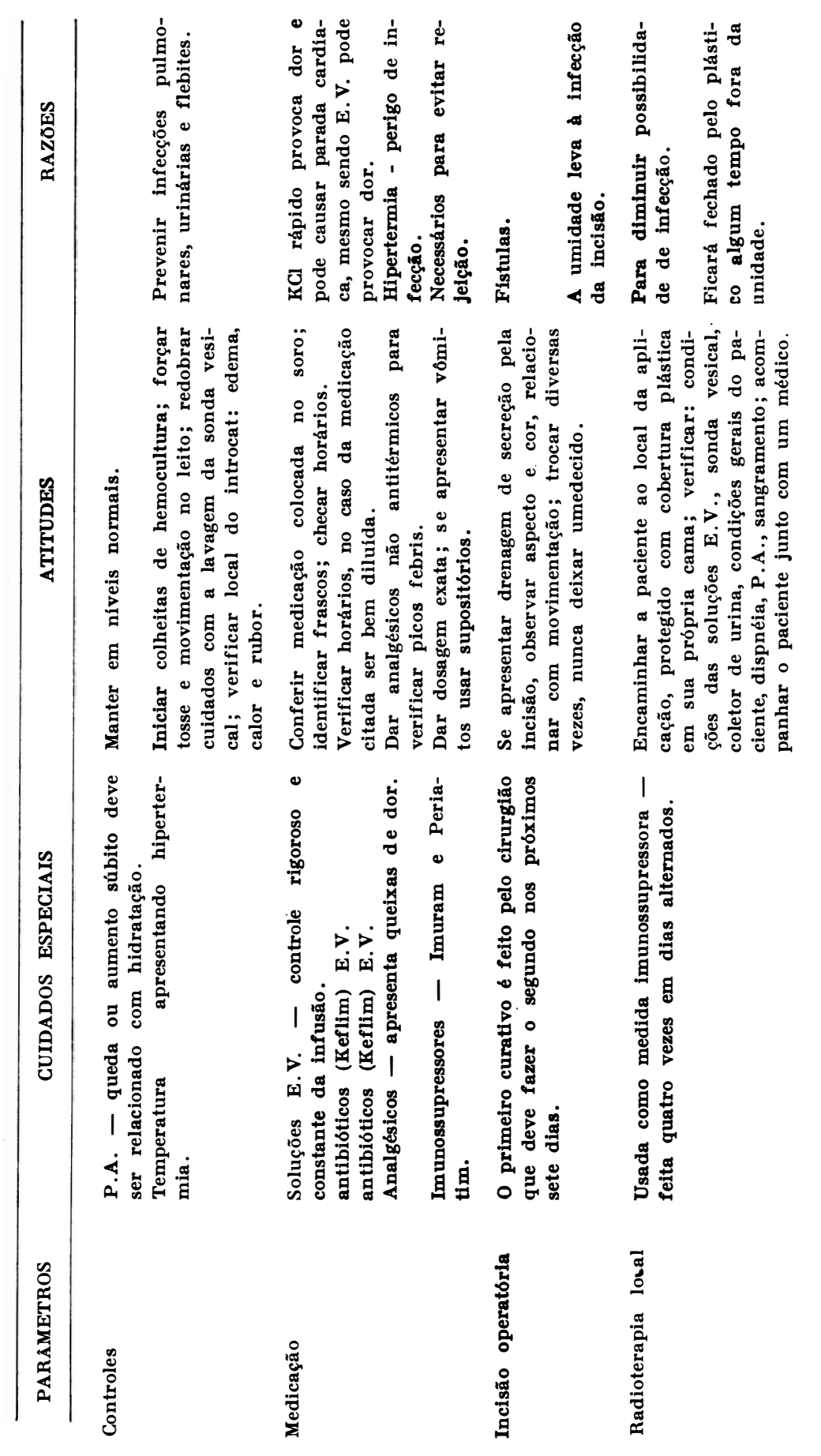




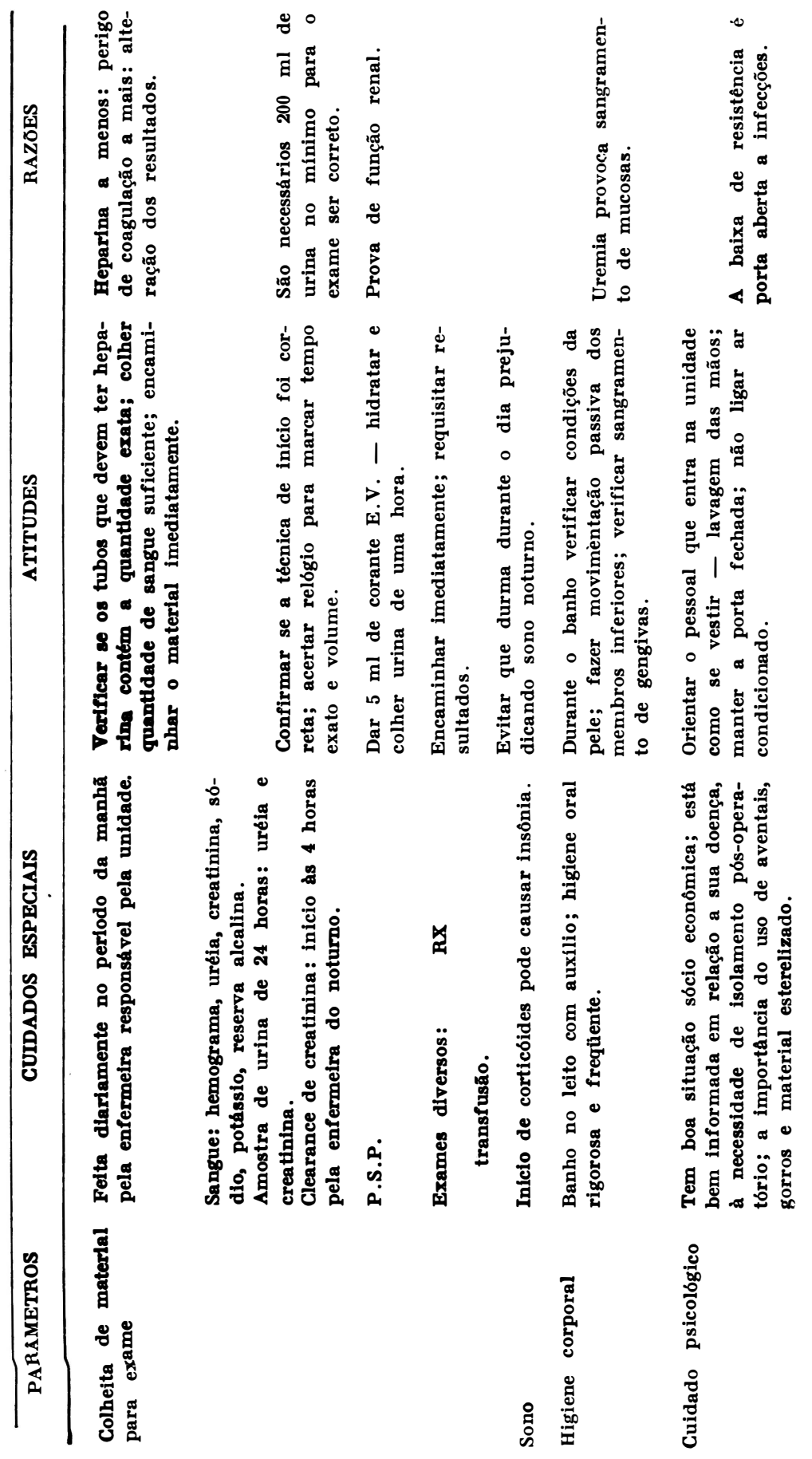




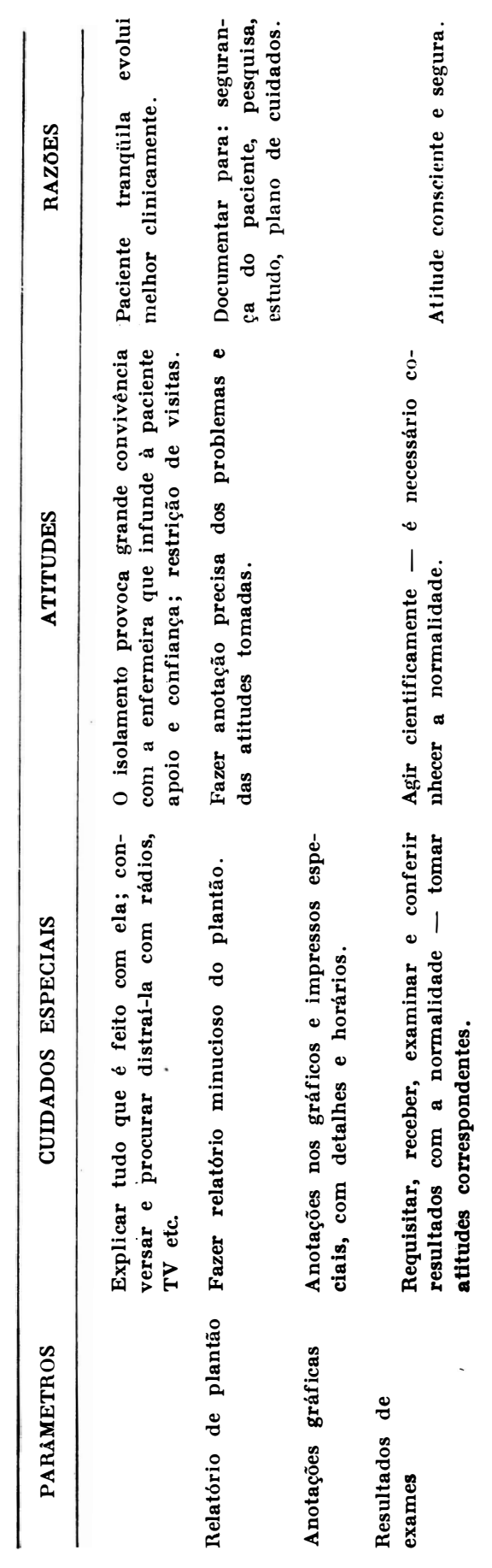

